\title{
Preparation and Characterization of Paclitaxel Loaded SF/PLLA-PEG-PLLA Nanoparticles via Solution-Enhanced Dispersion by Supercritical $\mathrm{CO}_{2}$
}

\author{
Zheng Zhao, ${ }^{1,2,3}$ Yi Li, $^{2}$ and Yu Zhang ${ }^{4}$ \\ ${ }^{1}$ State Key Laboratory of Advanced Technology for Materials Synthesis and Processing, Wuhan University of Technology, \\ Wuhan 430070, China \\ ${ }^{2}$ Institute of Textiles and Clothing, The Hong Kong Polytechnic University, Kowloon, Hong Kong \\ ${ }^{3}$ Biomedical Materials and Engineering Research Center of Hubei Province, Wuhan University of Technology, Wuhan 430070, China \\ ${ }^{4}$ Department of Mechanical Engineering, The Hong Kong Polytechnic University, Kowloon, Hong Kong
}

Correspondence should be addressed to Yi Li; tcliyi@polyu.edu.hk

Received 10 March 2015; Revised 31 May 2015; Accepted 17 June 2015

Academic Editor: Mircea Chipara

Copyright (C) 2015 Zheng Zhao et al. This is an open access article distributed under the Creative Commons Attribution License, which permits unrestricted use, distribution, and reproduction in any medium, provided the original work is properly cited.

Paclitaxel loaded silk fibroin/PLLA-PEG-PLLA (PTX-SF/PLLA-PEG-PLLA) nanoparticles with a mean particle size of about $651 \mathrm{~nm}$ were fabricated successfully by the SEDS process. Fourier transform infrared (FTIR) spectroscopy analysis indicated that the PTX was encapsulated by SF/PLLA-PEG-PLLA nanoparticles. X-ray powder diffraction (XRPD) analysis supported the results of FTIR analysis and also suggested that the crystalline state of PTX was decreased obviously. Furthermore, the UV-Vis/HPLC analysis showed that drug load (DL) and encapsulation efficiency (EE) were $18.1 \%$ and $90.2 \%$, respectively. The in vitro drug release experiment suggested that the PTX-SF/PLLA-PEG-PLLA nanoparticles exhibited a sustained release and only $16.1 \%$ and $24.5 \%$ of paclitaxel were released at $\mathrm{pH} 7.4$ and 6.0, respectively, in one week. The PTX-SF/PLLA-PEG-PLLA nanoparticles drug delivery system with $\mathrm{pH}$-dependent release property has potential application in the field of tumor therapy.

\section{Introduction}

Paclitaxel (PTX), a mitotic inhibitor used in cancer chemotherapy, has exhibited its potency against many kinds of cancers, including advanced ovarian, lung, and breast cancers $[1,2]$. The anticancer mechanism of PTX involves binding to microtubules, forming dysfunctional microtubules, and thus resulting in cell death [3]. However, its poor solubility in water drastically limits its clinical application in its natural form. To enhance drug solubility, paclitaxel is commercially formulated at $6 \mathrm{mg} / \mathrm{mL}$ in a vehicle composed of $1: 1$ blend of Cremophor EL and ethanol to make Taxol. Unfortunately, Cremophor EL is toxic and can cause severe sideeffects, including hypersensitivity reactions, nephrotoxicity, and neurotoxicity $[4,5]$. Also, the anticancer drug cannot be selectively accumulated into tumors tissues. The nonspecific distribution will reduce bioavailability and therapeutic efficiency of the anticancer drug because the drug spreads randomly during systemic circulation and effects cells (healthy or tumor cells) indiscriminately [6].

In order to improve the therapeutic efficacy of PTX and reduce side-effects, many researchers have developed alternative drug delivery systems, including parenteral emulsions, liposomes, and nanoparticles [7-10]. Among them, polymer nanoparticle drug delivery systems have attracted much attention owing to high bioavailability and sustained drug release characteristics $[11,12]$. Besides, it enables many different routes of drug administration, including injection, transdermal absorption, and oral application and inhalation. In particular, nanoparticles can extravasate into the tumor tissue via the leaky vessels surrounding tumors and then preferentially accumulate in solid tumors due to the enhanced permeation and retention (EPR) effect [13].

Suitable polymer should not only be biodegradable in response to biological condition but also exhibit excellent biocompatibility in human body. Biodegradable polymer can 
be divided into natural and synthetic biodegradable polymer. Natural biodegradable polymer such as silk fibroin (SF) has been widely used in biomedical fields owing to its unique properties, including good biocompatibility, biodegradability, and low inflammatory response [14-16]. Recently, SF nanoparticles have been utilized to load anticancer drug PTX $[17,18]$. However, natural polymer suffers from the high natural variability and the difficulties in processing.

Synthetic biodegradable polymers such as poly(L,Llactide) and its copolymers with polyethylene glycol (PEG) with precise chemical composition can overcome the disadvantages of silk fibroin above and are more easily designed for specific application, such as controlled rates of dissolution, permeability, degradation, and erosion [19-22]. Therefore, it has been commonly used as drug carrier in biomedical fields. However, the main disadvantage of these synthetic polymers is undesirable biological responses to cells owing to high crystallinity, strong hydrophobicity, and lack of bioactive functions [23].

The combination of a synthetic polymer with natural polymer especially silk fibroin can overcome their disadvantages and is an effective strategy to fabricate novel nanoparticles as carrier for PTX with the desired physical and chemical properties, mechanical strength, and biological responses. Most importantly, silk fibroin has many bioactive groups, which can provide sites for conjugating with tumor-specific ligands to achieve tumor-targeted drug delivery.

In our previous study, natural silk fibroin (SF) has been used to modify synthetic poly(l-lactide)-poly(ethylene glycol)-poly(l-lactide) (PLLA-PEG-PLLA) to obtain SF/ PLLA-PEG-PLLA composite nanoparticles prepared via solution-enhanced dispersion by supercritical $\mathrm{CO}_{2}$ (SEDS) [23]. In the SEDS process, the supercritical $\mathrm{CO}_{2}\left(\mathrm{scCO}_{2}\right)$ acts as antisolvent. In addition, the high velocity of the $\mathrm{scCO}_{2}$ breaks up the polymer solution into very small droplets and enhances mass transfer between the $\mathrm{scCO}_{2}$ and the droplets, instantaneously, resulting in phase separation and supersaturation of the polymer solution, thus leading to nucleation and precipitation of the polymer particle [24-26]. The incorporation of silk fibroin into the PLLA-PEG-PLLA nanoparticles improved biocompatibility and provided chemical modification. Moreover, the SF/PLLA-PEG-PLLA nanoparticles could be internalized by cells effectively [23]. Therefore, using the silk fibroin/PLLA-PEG-PLLA nanoparticles to load drugs and form a nanoparticle drug delivery system may be a potential application in the field of biomedical engineering.

In this study, the SEDS process was used to prepare a PTX loaded SF/PLLA-PEG-PLLA (PTX-SF/PLLA-PEGPLLA) nanoparticles delivery system. The surface morphology, physical and chemical properties, drug load, encapsulation efficiency, and in vitro drug release properties of PTXSF/PLLA-PEG-PLLA nanoparticles were measured.

\section{Materials and Methods}

2.1. Materials. Paclitaxel (PTX) was purchased from the Jiangsu Nanjing Zelang Medicine Technology Co., Ltd. (China). $\mathrm{CO}_{2}$ with a purity of $99.9 \%$ was supplied by the Hong Kong Specialty Gases Co., Ltd. (Hong Kong). The solvent,
Dichloromethane (DCM), was purchased from the Advanced Technology \& Industrial Co., Ltd. (Hong Kong). PLLAPEG-PLLA triblock polymer (MW $100 \mathrm{kDa}$, PEG 10\%) was purchased from Department of Medical Polymer Shandong Institute (Jinan, China). All other compounds were of analytical purity.

\subsection{Methods}

2.2.1. Preparation of SF/PLLA-PEG-PLLA Nanoparticles and PTX Loaded SF/PLLA-PEG-PLLA Nanoparticles by the SEDS Process. The SF/PLLA-PEG-PLLA composite nanoparticles can be prepared by the SEDS process given in [23]. For the preparation of PTX loaded SF/PLLA-PEG-PLLA (PTXSF/PLLA-PEG-PLLA) composite nanoparticles, the method was described as follows. Briefly, PTX and PLLA-PEGPLLA were dissolved in dichloromethane (DCM) solution together. Then water insoluble silk fibroin nanoparticles were dispersed into DCM solution to form suspension (for weight, PTX : silk fibroin nanoparticles : PLLA-PEG-PLLA = $5: 4: 16)$, and the final solution concentration of PTX-SF/ PLLA-PEG-PLLA was $0.5 \%(\mathrm{w} / \mathrm{v})$. Figure 1 shows a schematic diagram of the SEDS apparatus for preparation of the PTXSF/PLLA-PEG-PLLA nanoparticles, which consists of three major components: a $\mathrm{CO}_{2}$ supply system, a particle suspension delivery system and a high pressure vessel. In the particle suspension delivery system, an "injector" was made from a stainless steel cylinder and a piston with an O-ring seal. Using a SEDS process, when the desired pressure and temperature were stabilized, the suspension was injected through a stainless steel coaxial nozzle into the high-pressure vessel. The pressure, temperature, the flow rates of supercritical $\mathrm{CO}_{2}$, and the flow rates of suspension were kept at $10 \mathrm{MPa}, 35^{\circ} \mathrm{C}$; $25 \mathrm{NL} \cdot \mathrm{h}^{-1}$, and $0.5 \mathrm{~mL} \cdot \mathrm{min}^{-1}$, respectively.

2.2.2. Surface Morphology and Particle Size Distribution. The surface morphology of samples was visualized by a field emission scanning electron microscopy (FE-SEM, JEOL, JSM6490, Japan). Before analysis, samples were attached onto a carbon paint and then placed on an aluminum sample holder. The samples were made conductive by sputtering a thin layer of gold onto their surface. The particle size (PS) and particle size distribution (PSD) of PTX-SF/PLLA-PEG-PLLA nanoparticles dispersed in ethanol by sonication were measured by a laser diffraction particle size analyzer with a liquid module (LS 13320, Beckman Coulter, USA).

2.2.3. FTIR Analysis. The samples were combination with potassium bromide. Then the mixtures of the samples and potassium bromide were pressed into a transparent tablet. The FTIR spectra for the samples were recorded on an FTIR Perkin Elmer 1720 (Perkin Elmer, USA) in the transmission mode with the wave number ranging from 4,000 to $400 \mathrm{~cm}^{-1}$.

2.2.4. XRPD Analysis. Powder X-ray diffraction (XRPD) was performed by an X-ray diffractometer with a $\mathrm{Cu} K \alpha(\lambda=$ $1.5405 \AA$ ) radiation (D8 Advance, Bruker AXS, Germany). The measurement was carried out in a $2 \theta$ range of $5 \sim 45^{\circ}$ with 


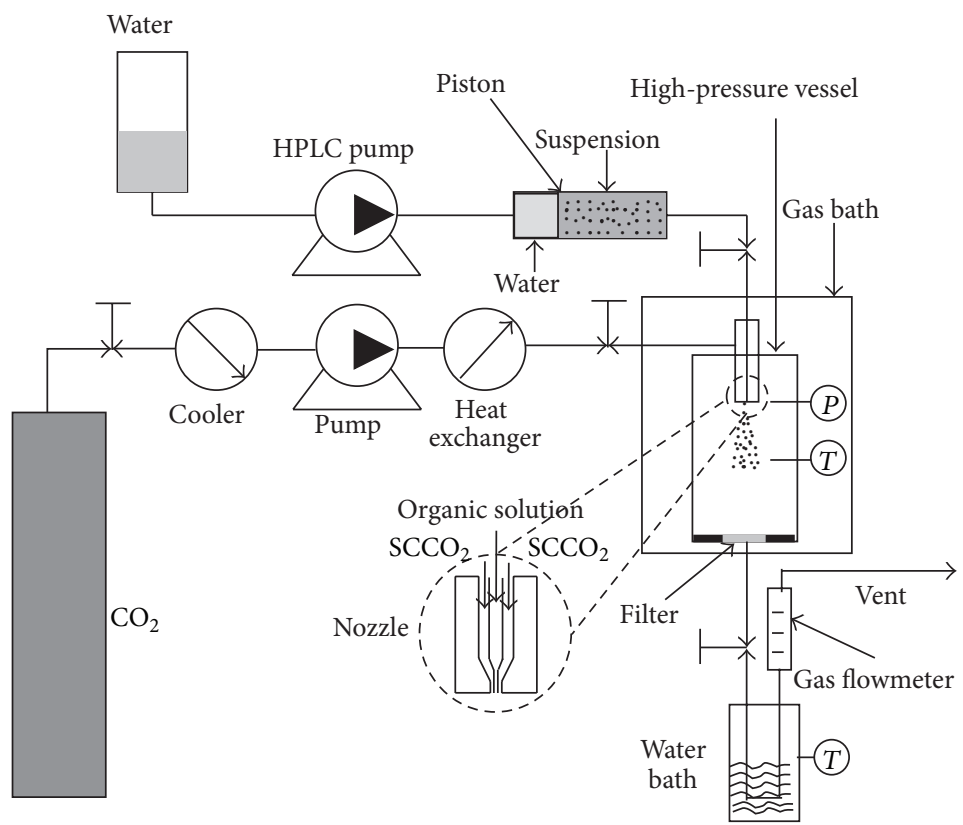

FIGURE 1: The schematic diagram of the SEDS apparatus for preparation of silk fibroin/PLLA-PE-PLLA composite nanoparticles.

$0.02^{\circ}$ step size and $10^{\circ} \mathrm{min}^{-1}$ scan speed with a $2 \mathrm{D}$ detector at $40 \mathrm{kV}$ and $40 \mathrm{~mA}$.

2.2.5. Drug Load (DL) and Encapsulation Efficiency (EE). The contents of PTX were analyzed by UV-Vis/HPLC (Waters $1100)$ system with the following conditions: stationary phase: reverse-phase column $(4.6 \mathrm{~mm} \times$ Aligent Eclipse $\mathrm{XDB}-\mathrm{C} 18$, $4.6 \mathrm{~mm} \times 250 \mathrm{~mm}$ internal diameter, pore size $5 \mu \mathrm{m})$; mobile phase: mixture of acetonitrile and water $(50: 50 \mathrm{v} / \mathrm{v})$; elution flow rate: $1 \mathrm{~mL} / \mathrm{min}$; detection wavelength: $227 \mathrm{~nm}$. All the solvent and distilled water used for aqueous solutions and buffer should be filtered via a $0.22 \mu \mathrm{m}$ membrane filter before use. The content of PTX in the samples was measured based on the peak area at the retention time. A calibration curve of standard PTX dissolving in mixture of acetonitrile and water $(50: 50 \mathrm{v} / \mathrm{v})$ was utilized to calculate the content of PTX in the samples. All the samples were conducted in triplicate.

The actual content of paclitaxel (PTX) in PTX-SF/PLLAPEG-PLLA nanoparticles can be determined by dissolving PTX-SF/PLLA-PEG-PLLA nanoparticles (3.0 mg accurately weighed) in $1 \mathrm{~mL}$ of DCM, and then $3 \mathrm{~mL}$ of an acetonitrile/water $(50: 50 \mathrm{v} / \mathrm{v})$ mixture was added. After being completely mixed, the solution was filtered and then a nitrogen stream was introduced to volatilize DCM under stirring at room temperature until a clear solution was obtained. The clear solution was put into a vial for UV-Vis/HPLC assay according to calibration curve. The theoretical drug content, the actual drug load, and encapsulation efficiency were calculated by (1), (2), and (3), respectively. Each experiment was carried out in independent triplicate:

$$
\text { Theoretical drug load }=\frac{W_{1}}{W_{2}} \times 100 \% \text {, }
$$

$$
\begin{array}{r}
\text { Actual drug load }=\frac{W_{3}}{W_{2}} \times 100 \%, \\
\text { Encapsulation efficiency }=\frac{W_{3}}{W_{1}} \times 100 \% .
\end{array}
$$

$W_{1}$ is the weight of theoretical PTX in the PTX-SF/PLLAPEG-PLLA nanoparticles; $W_{2}$ is the gross weight of the PTXSF/PLLA-PEG-PLLA nanoparticles; $W_{3}$ is the weight of the actual PTX encapsulated in the PTX-SF/PLLA-PEG-PLLA nanoparticles.

2.2.6. In Vitro Drug Release. Approximately $2 \mathrm{mg}$ of PTXSF/PLLA-PEG-PLLA nanoparticles was put into the pretreated dialysis bag, and then the bag was placed into a bottle with $100 \mathrm{~mL}$ of PBS (pH 7.4) and incubated in a water-bath shaker at $37^{\circ} \mathrm{C}$ and $60 \mathrm{rpm}$. Ten milliliters of solution was periodically removed and replaced by fresh $\mathrm{PBS}(\mathrm{pH}$ 7.4). The released drug was analyzed by UV-Vis/HPLC assay. As a control, an in vitro drug release experiment using free drug (of equivalent weight to that in the drug-loaded nanoparticles) was performed in similar conditions. The in vitro drug release curves were drawn based on the cumulative release percentage of PTX $(\%, w / w)$ in PBS solution over long periods of time.

\section{Results and Discussion}

3.1. Surface Morphology and Structure of PTX-SF/PLLA-PEGPLLA Nanoparticles. Figures 2(a) and 2(b) show the FE-SEM photographs of SF/PLLA-PEG-PLLA and PTX-SF/PLLAPEG-PLLA nanoparticles obtained by the SEDS process, respectively. The particle size distribution of the PTXSF/PLLA-PEG-PLLA nanoparticles is shown in Figure 3. According to our previous study, the SF/PLLA-PEG-PLLA 


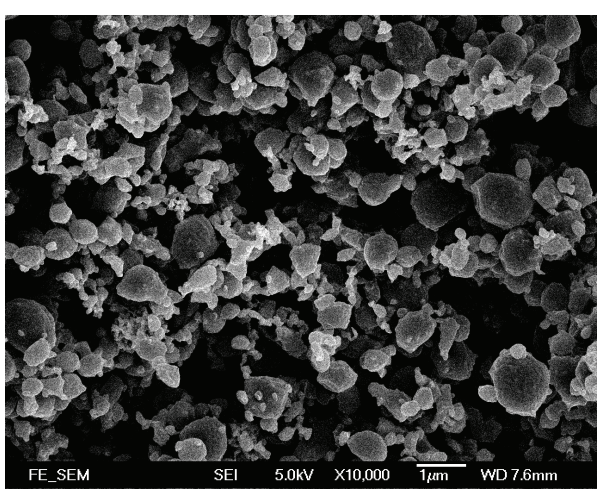

(a)

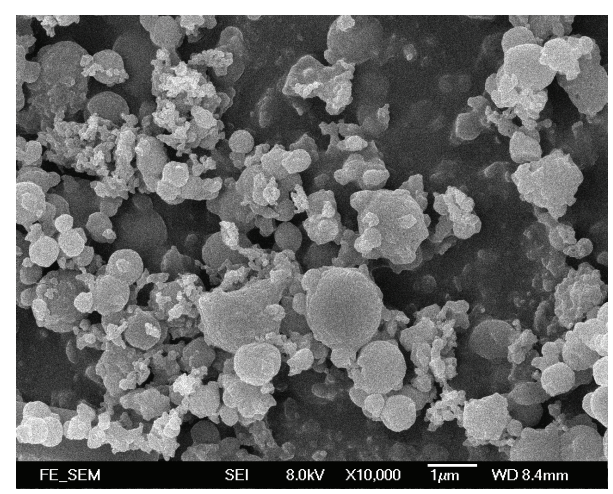

(b)

FIGURE 2: FE-SEM photographs of SF/PLLA-PEG-PLLA nanoparticles (a) and PTX-SF/PLLA-PEG-PLLA nanoparticles (b) obtained by the SEDS process.

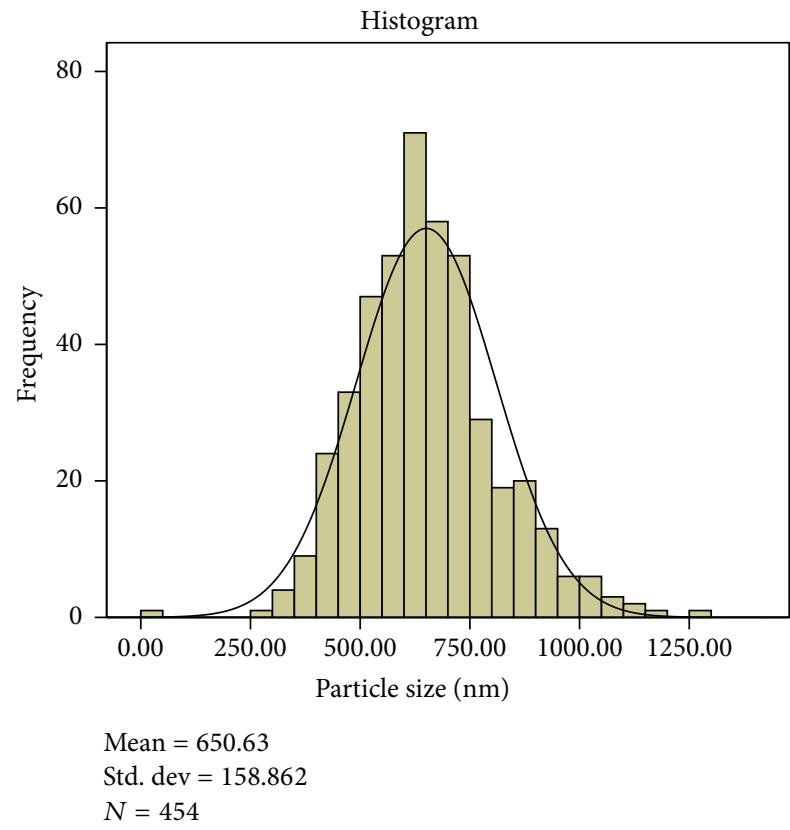

FIgure 3: Particle size distribution of PTX-SF/PLLA-PEG-PLLA obtained by the SEDS process.

nanoparticles exhibit a composite structure with mean particle size of $634 \mathrm{~nm}$ and silk fibroin wrapped with PLLAPEG-PLLA triblock polymer [23]. According to Figures 2(b) and 3, the PTX-SF/PLLA-PEG-PLLA nanoparticles possess a spherical shape with a mean particle size of about $651 \mathrm{~nm}$ and particle size distribution is 0.635 . It suggested that the SEDS process is an effective method to prepare SF/PLLAPEG-PLLA and PTX-SF/PLLA-PEG-PLLA nanoparticles.

3.2. FTIR Analysis. Figure 4 shows FTIR spectra of free PTX, physical mixtures of SF and PLLA-PEG-PLLA (SF+PLLA-PEG-PLLA), SF/PLLA-PEG-PLLA, and PTXSF/PLLA-PEG-PLLA prepared by the SEDS process. The characteristic absorption peaks at $1759 \mathrm{~cm}^{-1}$ are attributed to

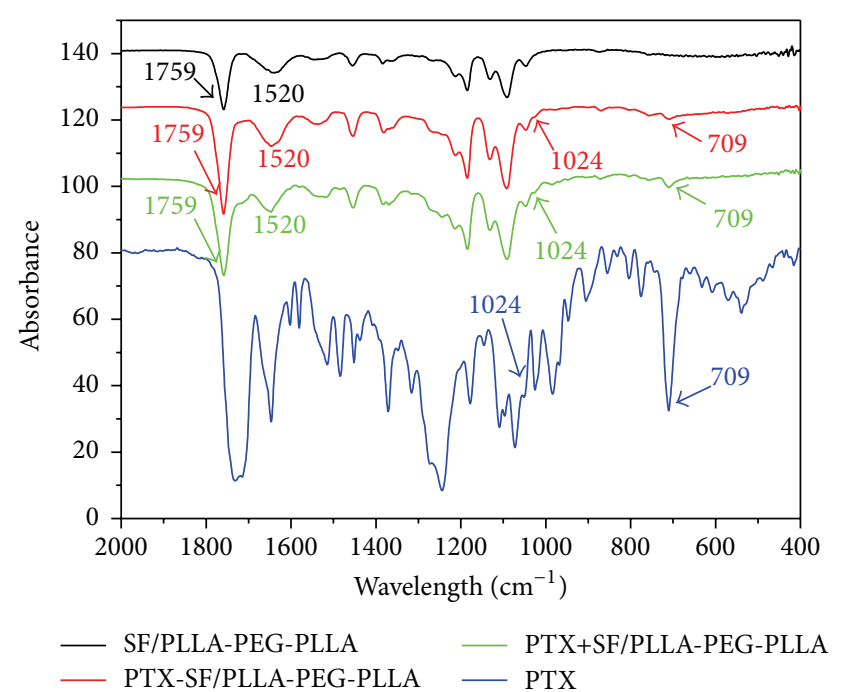

FIGURE 4: FTIR spectra of PTX, SF/PLLA-PEG-PLLA nanoparticles, physical mixture of PTX and SF/PLLA-PEG-PLLA nanoparticles, and PTX-SF/PLLA-PEG-PLLA nanoparticles.

the $\mathrm{C}=\mathrm{O}$ stretching in PLLA-PEG-PLLA. The characteristic peak of amide II at $1520 \mathrm{~cm}^{-1}$ is assigned to the $\beta$-sheet of silk fibroin. The major characteristic absorption peak of $\mathrm{C}-\mathrm{C}$ stretching is shown for crystalline PTX at $709 \mathrm{~cm}^{-1}$. After the SEDS process, the main peaks $\left(709 \mathrm{~cm}^{-1}\right)$ appear at the same position compared with the physical mixtures, which suggests that the resulting products contain PTX. The presence of the peak at $1024 \mathrm{~cm}^{-1}$ further demonstrates that PTX has been encapsulated by SF/PLLA-PEG-PLLA nanoparticles successfully.

3.3. XRPD Analysis. To investigate the physical state of the drug-loaded polymer nanoparticles prepared by the SEDS process, XRPD analysis was performed on the drug-loaded polymer nanoparticles. Figure 5 shows XRPD patterns of free PTX, physical mixtures of SF and PLLA-PEG-PLLA 


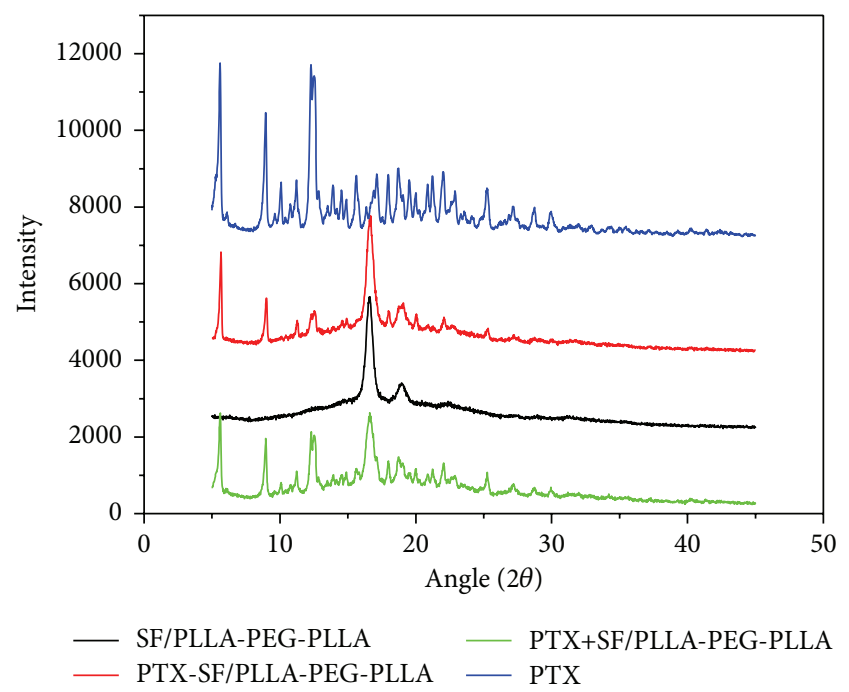

Figure 5: XRPD patterns of free PTX, SF/PLLA-PEG-PLLA, and PTX-SF/PLLA-PEG-PLLA prepared by the SEDS process.

(SF+PLLA-PEG-PLLA), SF/PLLA-PEG-PLLA, and PTXSF/PLLA-PEG-PLLA prepared by the SEDS process. Original paclitaxel exhibited the main characteristic XRPD peaks at $2 \theta$ $=5.6^{\circ}, 9.1^{\circ}, 10.1^{\circ}, 12.7^{\circ}, 15.6$, and $25.3^{\circ}$. After the SEDS process, nearly all the main characteristic peaks of crystalline PTX became weak, especially peaks at $2 \theta=10.1^{\circ}$ and 15.6 . In the physical mixtures, the weak characteristic peaks of crystalline PTX at $10.1^{\circ}$ can still be observed in the XRD patterns. This indicated that the SEDS process reduces the crystallinity of PTX obviously.

3.4. Drug Load (DL) and Encapsulation Efficiency (EE). DL and $\mathrm{EE}$ are two kinds of important parameters to determine the drug loading properties of nanoparticles. In the present study, the theoretical weight ratio of drug to polymer was selected as 1:4 (i.e., theoretical DL of 20\%). The results of HPLC assay indicate the DL and EE are $18.1 \%$ and $90.2 \%$, respectively. Many researchers have studied preparation of PTX loaded polymer nanoparticles by supercritical antisolvent process. Kang et al. prepared PTX-PLLA microparticles and found the theoretical DL and DL and EE of PTX-PLLA microparticles were $20 \%, 14.33 \%$, and $62.68 \%$, respectively [27]. Lee et al. also obtain PTX-PLLA particles. The results indicated that when the theoretical DL ranges from $3 \%$ to $10 \%$, DD and EE are $1.98 \sim 7.87 \%$ and $59.3 \% \sim 78.7 \%$, respectively [28]. Li et al. studied the effect of process parameters on coprecipitation of PTX and PLLA by supercritical antisolvent process and found that DL was influenced by different process parameters such as solvent, the concentration of solute, and flow rate of solution [29]. Besides, Ouyang et al. fabricated the PTX-PLA-PEG-PLA microparticles by similar technique. When theoretical DL is $20 \%$, DL ranges from $5.1 \%$ to $7.1 \%$ for different PEG contents, and EE ranges from $25.5 \%$ to $35.5 \%$ [30]. The different DL and EE may result from the different precipitation kinetics of the drug and polymer in the supercritical antisolvent process $[27,30]$. Further studies are

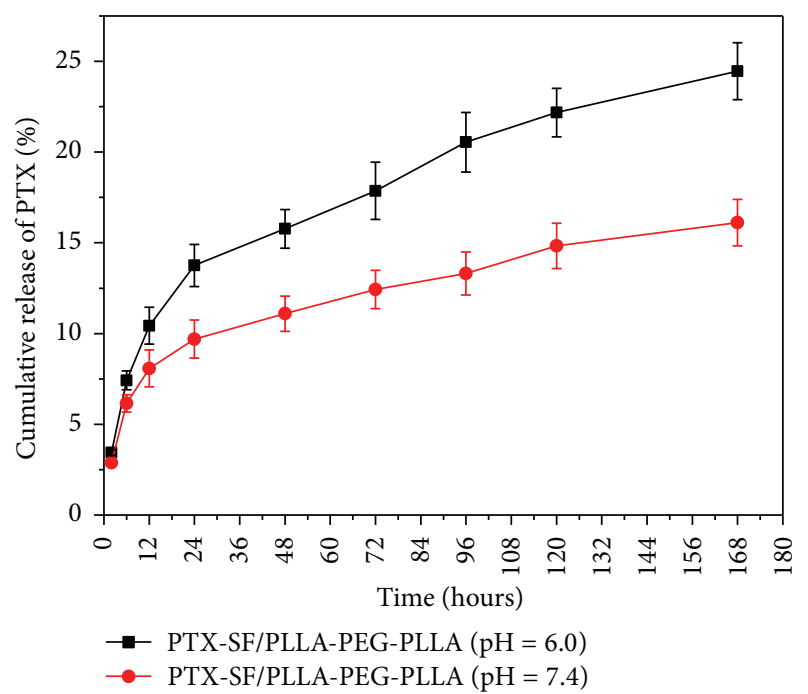

FIGURE 6: In vitro release profile of PTX from PTX-SF/PLLA-PEGPLLA nanoparticles in PBS buffer ( $\mathrm{pH} 6.0$ and 7.4).

demanded to explore the actual reason. As nearly all the drug is loaded on the SF/PLLA-PEG-PLLA nanoparticles, it would be commercially attractive.

3.5. In Vitro Drug Release. In terms of the unique microenvironment surrounding tumor cells, in which the average extracellular $\mathrm{pH}$ value in tumors is between 6.0 and 7.0, however, the extracellular $\mathrm{pH}$ value in normal tissues is about 7.4 .

The in vitro release profile of PTX from nanoparticles was studied in different $\mathrm{pH}$ conditions ( $\mathrm{pH} 6.0$ and 7.4). Figure 6 shows the in vitro release profile of PTX from PTX loaded SF/PLLA-PEG-PLLA (PTX-SF/PLLA-PEG-PLLA) nanoparticles in PBS buffer ( $\mathrm{pH} 6.0$ and 7.4). As shown in Figure 5, the drug release in PBS buffer ( $\mathrm{pH} 7.4)$ was slower than that in PBS buffer ( $\mathrm{pH} 6.0$ ). And $16.1 \%$ and $24.5 \%$ of paclitaxel were released at pH 7.4 and 6.0, respectively, in one week. Therefore, there was no burst effect and PTX can be released in a controlled way from SF/PLLA-PEG-PLLA nanoparticles. Furthermore, nanoencapsulation of PTX by SF/PLLA-PEGPLLA nanoparticles could improve the solubility of PTX.

The faster release of PTX from the PTX-SF/PLLA-PEGPLLA nanoparticles at lower $\mathrm{pH}$ conditions results from two possible reasons. One is that lower $\mathrm{pH}$ may cause faster degradation of SF/PLLA-PEG-PLLA polymer. The other is that the amino group of the drug could be protonated at lower $\mathrm{pH}$ condition. This $\mathrm{pH}$-dependent release property may be beneficial for tumor treatment $[31,32]$. It is likely that the release of PTX from SF/PLLA-PEG-PLLA nanoparticles within healthy physiological $\mathrm{pH}$ levels $(\mathrm{pH}$ 7.4) is slow, sustained, and relatively suppressed during systemic circulation. When SF/PLLA-PEG-PLLA nanoparticles were delivered into the location of solid tumor, an enhanced release of PTX from nanoparticles could be triggered by the mildly acidic tumor microenvironment ( $\mathrm{pH}$ 6.0) to ensure that the intracellular drug concentration reaches the therapeutic dose. 
Therefore, $\mathrm{pH}$-dependent release property may decrease the side-effect of PTX to healthy cells owing to relatively low PTX concentration and enhance the therapeutic efficiency against tumor cells.

\section{Conclusions}

PTX-SF/PLLA-PEG-PLLA nanoparticles with mean particle size of $651 \mathrm{~nm}$ were prepared by the SEDS process successfully. FTIR and XRPD analysis indicated PTX was encapsulated by SF/PLLA-PEG-PLLA nanoparticles. Furthermore, the PTX became more amorphous after the SEDS process. The PTX-SF/PLLA-PEG-PLLA nanoparticles exhibited a controlled drug release. In particular the drug release rate in PBS solution could be accelerated with decrease of $\mathrm{pH}$ value from 7.4 to 6.0 . This $\mathrm{pH}$-dependent release property may decrease the side-effect of PTX to healthy cells and enhance the therapeutic efficiency against tumor cells due to the acidic tumor microenvironment. In terms of these characteristics, PTX-SF/PLLA-PEG-PLLA nanoparticles have potential application in the field of tumor therapy.

\section{Conflict of Interests}

The authors declare that there is no conflict of interests regarding the publication of this paper.

\section{Acknowledgments}

The authors would like to thank the Hong Kong Research Grant Council and the Hong Kong Polytechnic University through Projects PolyU5242/09E and G-YM63. Also, the authors would like to thank the support of Natural Science Foundation of Hubei Province through Project 2014CFB839, Doctoral Research Fund of Wuhan University of Technology through Project 471-40120093, Opening Project KJS1415 of National Engineering Laboratory for Modern Silk, and Guangdong Provincial Department of Science and Technology through Projects 2012B050800002 and 2012B091000143.

\section{References}

[1] H.-Y. Cho, C. K. Lee, and Y.-B. Lee, "Preparation and evaluation of PEGylated and folate-PEGylated liposomes containing paclitaxel for lymphatic delivery," Journal of Nanomaterials, vol. 2015, Article ID 471283, 10 pages, 2015.

[2] F. Danhier, P. Danhier, C. J. De Saedeleer et al., "Paclitaxelloaded micelles enhance transvascular permeability and retention of nanomedicines in tumors," International Journal of Pharmaceutics, vol. 479, no. 2, pp. 399-407, 2015.

[3] G. Ruan and S.-S. Feng, "Preparation and characterization of poly(lactic acid)-poly(ethylene glycol)-poly(lactic acid) (PLAPEG-PLA) microspheres for controlled release of paclitaxel," Biomaterials, vol. 24, no. 27, pp. 5037-5044, 2003.

[4] F. Danhier, N. Lecouturier, B. Vroman et al., "Paclitaxel-loaded PEGylated PLGA-based nanoparticles: in vitro and in vivo evaluation," Journal of Controlled Release, vol. 133, no. 1, pp.11-17, 2009.
[5] P.-P. Lv, W. Wei, H. Yue, T.-Y. Yang, L.-Y. Wang, and G.-H. Ma, "Porous quaternized chitosan nanoparticles containing paclitaxel nanocrystals improved therapeutic efficacy in non-smallcell lung cancer after oral administration," Biomacromolecules, vol. 12, no. 12, pp. 4230-4239, 2011.

[6] A. K. Singla, A. Garg, and D. Aggarwal, "Paclitaxel and its formulations," International Journal of Pharmaceutics, vol. 235, no. 1-2, pp. 179-192, 2002.

[7] T. Yang, M.-K. Choi, F.-D. Cui et al., "Preparation and evaluation of paclitaxel-loaded PEGylated immunoliposome," Journal of Controlled Release, vol. 120, no. 3, pp. 169-177, 2007.

[8] R. T. Liggins and H. M. Burt, "Paclitaxel-loaded poly (L-lactic acid) microspheres 3: blending low and high molecular weight polymers to control morphology and drug release," International Journal of Pharmaceutics, vol. 282, no. 1-2, pp. 61-71, 2004.

[9] Z. Zhang and S.-S. Feng, "The drug encapsulation efficiency, in vitro drug release, cellular uptake and cytotoxicity of paclitaxelloaded poly(lactide)-tocopheryl polyethylene glycol succinate nanoparticles," Biomaterials, vol. 27, no. 21, pp. 4025-4033, 2006.

[10] K. M. Huh, H. S. Min, S. C. Lee, H. J. Lee, S. Kim, and K. Park, “A new hydrotropic block copolymer micelle system for aqueous solubilization of paclitaxel," Journal of Controlled Release, vol. 126, no. 2, pp. 122-129, 2008.

[11] Y. B. Patil, U. S. Toti, A. Khdair, L. Ma, and J. Panyam, "Singlestep surface functionalization of polymeric nanoparticles for targeted drug delivery," Biomaterials, vol. 30, no. 5, pp. 859-866, 2009.

[12] Z. Keresztessy, M. Bodnár, E. Ber et al., "Self-assembling chitosan/poly- $\gamma$-glutamic acid nanoparticles for targeted drug delivery," Colloid and Polymer Science, vol. 287, no. 7, pp. 759$765,2009$.

[13] L. Brannon-Peppas and J. O. Blanchette, "Nanoparticle and targeted systems for cancer therapy," Advanced Drug Delivery Reviews, vol. 56, no. 11, pp. 1649-1659, 2004.

[14] Y. Lian, J.-C. Zhan, K.-H. Zhang, and X.-M. Mo, "Fabrication and characterization of curcumin-loaded silk fibroin/P(LLACL) nanofibrous scaffold," Frontiers of Materials Science, vol. 8, no. 4, pp. 354-362, 2014.

[15] B.-J. Dong and Q. Lu, "Conductive Au nanowires regulated by silk fibroin nanofibers," Frontiers of Materials Science, vol. 8, no. 1, pp. 102-105, 2014.

[16] Z. Zhao, Y. Li, and M. Xie, "Silk fibroin-based nanoparticles for drug delivery," International Journal of Molecular Sciences, vol. 16, no. 3, pp. 4880-4903, 2015.

[17] M. Chen, Z. Shao, and X. Chen, "Paclitaxel-loaded silk fibroin nanospheres," Journal of Biomedical Materials Research, Part A, vol. 100, no. 1, pp. 203-210, 2012.

[18] P. Wu, Q. Liu, R. Li et al., "Facile preparation of paclitaxel loaded silk fibroin nanoparticles for enhanced antitumor efficacy by locoregional drug delivery," ACS Applied Materials and Interfaces, vol. 5, no. 23, pp. 12638-12645, 2013.

[19] T. Deepak, D. Michel, and P. Yashwant, Nanoparticulate Drug Delivery Systems, Informa Healthcare USA, New York, NY, USA, 2007.

[20] B. Dhurai, N. Saraswathy, R. Maheswaran et al., "Electrospinning of curcumin loaded chitosan/poly (lactic acid) nanofilm and evaluation of its medicinal characteristics," Frontiers of Materials Science, vol. 7, no. 4, pp. 350-361, 2013. 
[21] C. G. Mothé, A. D. Azevedo, W. S. Drumond, S. H. Wang, and R. D. Sinisterra, "Preparation and characterization of poly(l,1-lactide)-b-poly(ethylene glycol)-b-poly(l,l-lactide) (PLLA-PEG-PLLA) microspheres having encapsulated tetracycline," Journal of Thermal Analysis and Calorimetry, vol. 106, no. 3, pp. 671-677, 2011.

[22] A.-Z. Chen, L. Li, S.-B. Wang et al., "Study of $\mathrm{Fe}_{3} \mathrm{O}_{4}$-PLLAPEG-PLLA magnetic microspheres based on supercritical $\mathrm{CO}_{2}$ : preparation, physicochemical characterization, and drug loading investigation," The Journal of Supercritical Fluids, vol. 67, pp. 139-148, 2012.

[23] Z. Zhao, Y. Li, Y. Zhang et al., "Development of silk fibroin modified poly(L-lactide)-poly(ethylene glycol)-poly(L-lactide) nanoparticles in supercritical $\mathrm{CO}_{2}$," Powder Technology, vol. 268, no. 1, pp. 118-125, 2014.

[24] A.-Z. Chen, L. Li, S.-B. Wang et al., "Nanonization of methotrexate by solution-enhanced dispersion by supercritical $\mathrm{CO}_{2}$," Journal of Supercritical Fluids, vol. 67, pp. 7-13, 2012.

[25] Z. Zhao, A. Z. Chen, Y. Li et al., "Fabrication of silk fibroin nanoparticles for controlled drug delivery," Journal of Nanoparticle Research, vol. 14, no. 4, article 736, 2012.

[26] Z. Zhao, Y. Li, A.-Z. Chen et al., "Generation of silk fibroin nanoparticles via solution-enhanced dispersion by supercritical $\mathrm{CO}_{2}$," Industrial \& Engineering Chemistry Research, vol. 52, no. 10, pp. 3752-3761, 2013.

[27] Y. Kang, J. Wu, G. Yin et al., "Characterization and biological evaluation of paclitaxel-loaded poly(L-lactic acid) microparticles prepared by supercritical $\mathrm{CO}_{2}$," Langmuir, vol. 24, no. 14, pp. 7432-7441, 2008.

[28] L. Y. Lee, C. H. Wang, and K. A. Smith, "Supercritical antisolvent production of biodegradable micro- and nanoparticles for controlled delivery of paclitaxel," Journal of Controlled Release, vol. 125, no. 2, pp. 96-106, 2008.

[29] W. Li, G. Liu, L. Li, J. Wu, Y. Lü, and Y. Jiang, "Effect of process parameters on co-precipitation of paclitaxel and poly(L-lactic acid) by supercritical antisolvent process," Chinese Journal of Chemical Engineering, vol. 20, no. 4, pp. 803-813, 2012.

[30] P. Ouyang, Y.-Q. Kang, G.-F. Yin, Z.-B. Huang, Y.-D. Yao, and X.-M. Liao, "Fabrication of hydrophilic paclitaxel-loaded PLA-PEG-PLA microparticles via SEDS process," Frontiers of Materials Science in China, vol. 3, no. 1, pp. 15-24, 2009.

[31] J. Liu, Y. Huang, A. Kumar et al., "PH-sensitive nano-systems for drug delivery in cancer therapy," Biotechnology Advances, vol. 32, no. 4, pp. 693-710, 2014.

[32] S. J. T. Rezaei, H. S. Abandansari, M. R. Nabid, and H. Niknejad, "PH-responsive unimolecular micelles self-assembled from amphiphilic hyperbranched block copolymer for efficient intracellular release of poorly water-soluble anticancer drugs," Journal of Colloid and Interface Science, vol. 425, pp. 27-35, 2014. 

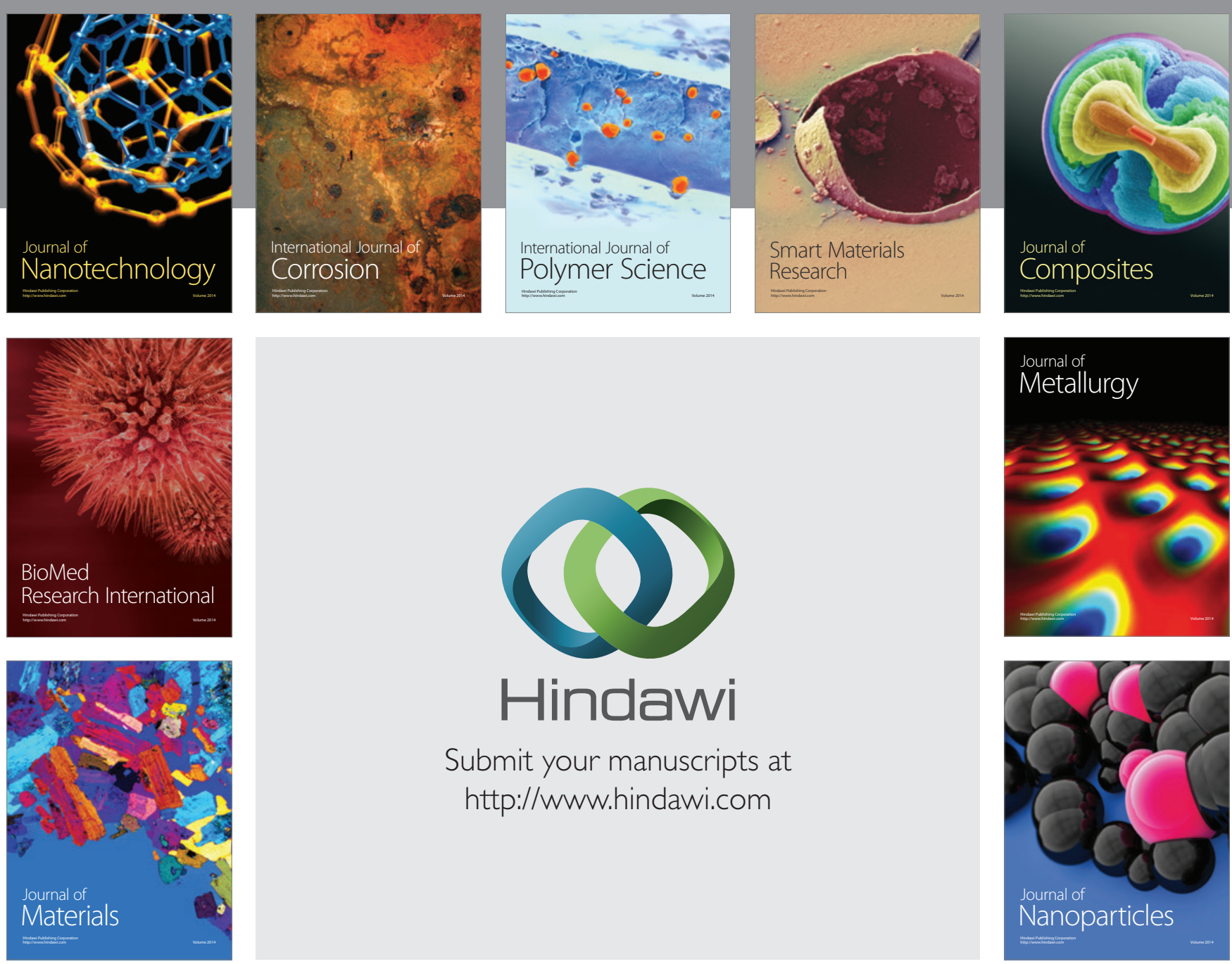

Submit your manuscripts at http://www.hindawi.com
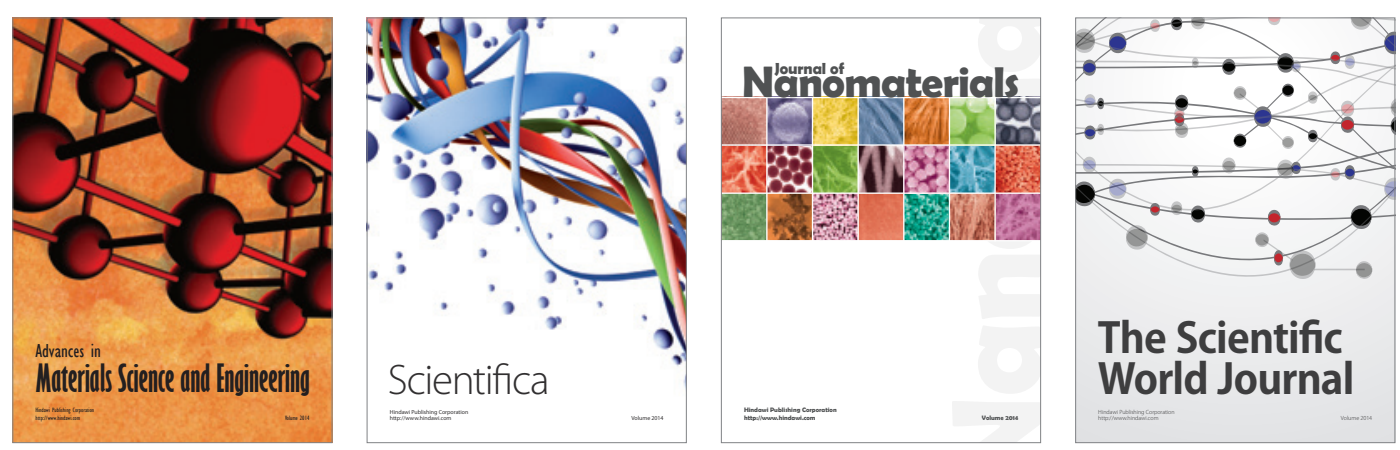

\section{The Scientific World Journal}
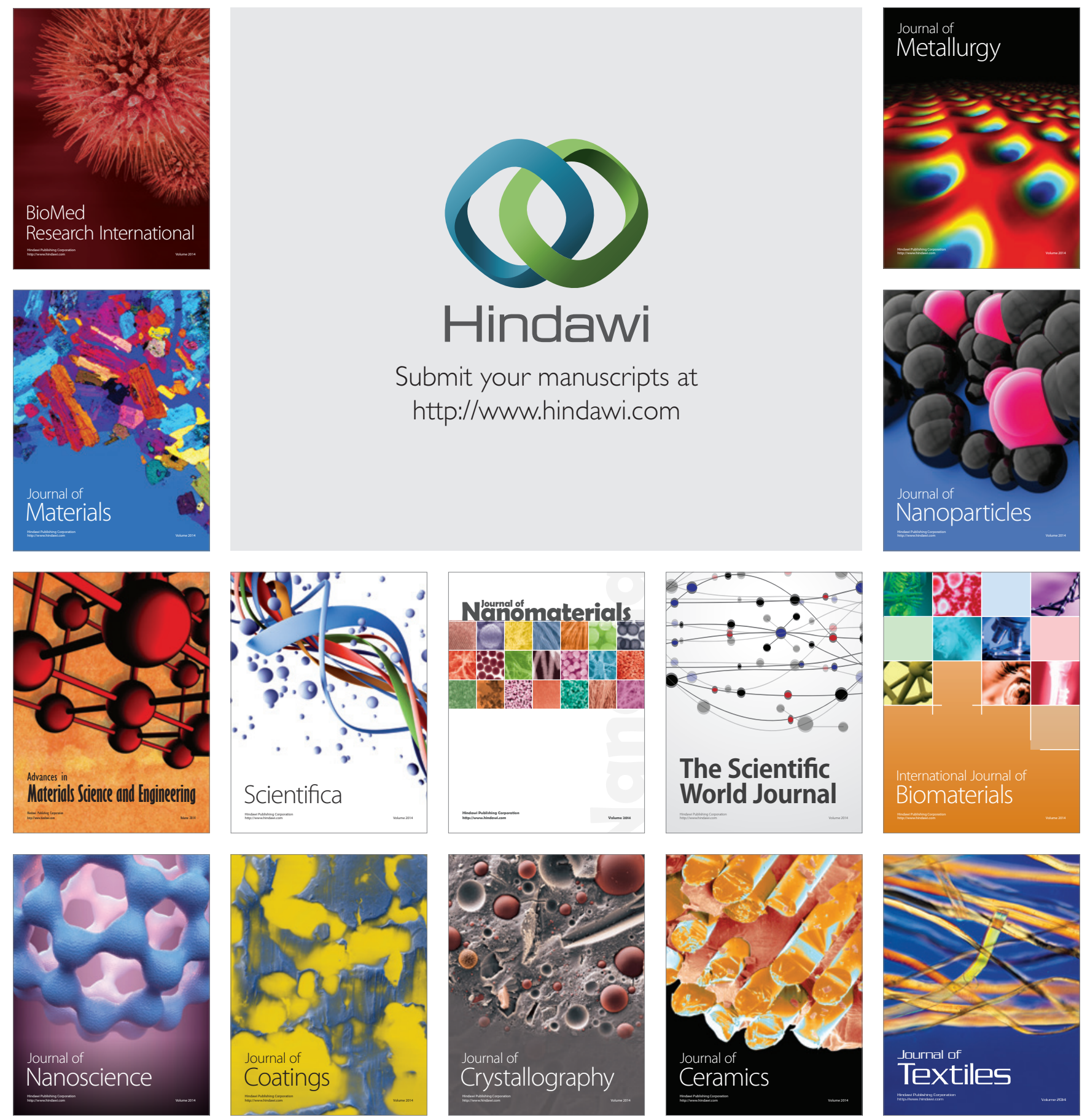